\title{
Fast Unbiased Echo Canceller Update During ADSL Transmission
}

\author{
Milos Milosevic*, Student Member, IEEE, Takao Inoue, Student Member, IEEE, Peter Molnar, \\ Member, IEEE, and Brian L. Evans, Senior Member, IEEE
}

\begin{abstract}
This paper presents an efficient method for updating echo canceller (EC) coefficients in ADSL transceivers during transmission that removes the dependency of updates on the far end signal. The new method improves on the frequency domain EC architecture by Ho, Cioffi, and Bingham in that it converges faster, improves the stability to perturbation, and is not prone to bias from pilot tones. The new EC updating system architecture is presented for both the central office and remote terminal systems and is fully compliant with the ITU-T G.DMT standard.
\end{abstract}

Index Terms - Echo suppression, least mean square methods, broadband communication, discrete multitone modulation.

\section{INTRODUCTION}

$\mathrm{D}$ iscrete Multi-Tone (DMT) is a multicarrier modulation method in which available bandwidth of twisted-pair copper media is divided into numerous subchannels or bins via a fast Fourier transform (FFT). Data simultaneously flows downstream from the central office transceiver to the remote terminal (customer transceiver), and upstream from the remote terminal to the central office. The DMT technique has been adopted in the US by the American National Standards Institute (ANSI) T1.413-1998 standard (Asymmetric Digital Subscriber Loop ADSL standard) [3] and internationally by the International Telecommunications Union G.DMT (G.992.1) [4] and G.Lite (G.992.2) [5] standards. In the ANSI standard, DMT is used to generate up to 250 separate $4.3125 \mathrm{kHz}$ wide downstream subchannels from $26 \mathrm{kHz}$ to 1.1 MHz. Likewise, DMT is used to generate 26 upstream subchannels from $26 \mathrm{kHz}$ to $138 \mathrm{kHz}$. Allowing the downstream transmission to use the same frequency band as the upstream transmission increases the available downstream bandwidth and thereby enables a higher downstream data rate. In G.DMT, this band is situated at the lower end of the ADSL frequency allocation. Given the spectral characteristics of the twisted pair and the noise, the overlapped band is generally a

Manuscript received September 22, 2000.

${ }^{*}$ M, Milosevic (phone: 512-775-7115), T. Inoue and B. L. Evans are with the Dept. of Electrical and Computer Engineering, The University of Texas at Austin, Austin, TX 78712. E-mail: \{milos, inoue, bevans\}@ece.utexas.edu. M. Milosevic, T. Inoue and P. Molnar (rpmolnar@yahoo.com) were with Motorola during the course of this work. region of high signal-to-noise ratio (SNR) and is thus able to support a large number of bits.

Echo is the spectral leakage of the transmitted signal from the local transmitter into the local receiver where it interferes with the desired far-end signal. Echo occurs through a 4-to-2 wire analog interface called the hybrid. A hybrid cannot usually provide the high rejection level of the transmitted signal needed $(\sim 70 \mathrm{~dB})$ for cost reasons and variations encountered in loop lengths and configurations. Echo cancellation (EC) algorithms remove the echo signal and allow simultaneous transmission in the same frequency band in both the upstream and downstream directions.

ADSL systems employing the overlapped spectrum often have an EC training period at startup during which the characteristics of the Echo Channel Impulse Response (ECIR) are identified. More sophisticated systems not only perform training at startup, but also adapt the EC coefficients continually during transmission in order to track the changes in the ECIR over time (heating up of the hybrid circuitry is a common cause of the change in the ECIR). Fast and reliable EC coefficient updating mechanism is a valuable tool that allows an overlapped ADSL transmission system to maintain link integrity.

We propose an improved echo canceller update system architecture that is amenable to VLSI implementation. The new architecture removes the effects of the far-end signal from the LMS-based updates of the echo canceller taps. The proposed architecture exhibits fast convergence, stability to perturbation, and resistance to bias errors because it is independent of the far-end signal. Section II reviews the echo canceller architecture of Ho, Cioffi, and Bingham [1]. Section III presents the proposed architecture for both the remote terminal and central office. Section IV shows experimental results. Section V concludes the paper

\section{CONVENTIONAL ARCHITECTURE}

Cioffi and Bingham [2] propose data-driven echo cancellation architecture for ADSL based entirely in the time domain. Later, building on that work, Ho, Cioffi, and Bingham [1] propose a high-speed method for echo cancellation in DMT systems during transmission. The architecture in [1] requires 93\% fewer multiplications than the DMT echo canceller in [2] but $50 \%$ more iterations to converge. The method in [1] cancels the echo directly in the frequency domain and uses time domain processing only to make the echo signal appear 
periodic, which avoids costly fully time-domain echo cancellation. Ho et al. model the ECIR as a finite impulse response (FIR) filter and model the echo as a linear convolution of the sequence of transmitted symbols and the ECIR. A symbol is a grouping of time samples consisting of 544 samples in the downstream direction and 68 samples in the upstream direction. The EC removes echo by using knowledge of the transmitted symbol and an estimate of the ECIR.

The time-domain unit called circular echo synthesis (CES) unit changes the linear convolution of the ECIR and the locally transmitted symbol into their circular convolution. Circular convolution can be computed using the fast Fourier transform (FFT) block, which is already present as a part of the discrete multitone demodulation receiver. The circular convolution operation by CES presents a modified echo symbol at the input of the FFT that, at the output, can be modeled as a multiplication of the frequency domain representations of ECIR and the locally transmitted symbol. An estimate of the echo is synthesized in the frequency domain and subtracted from the modified symbol, which leaves a remainder: the far-end symbol plus residual echo. The residual echo component results from inaccuracies in the FIR model of the ECIR and/or from changes in the ECIR due to changes in physical conditions (e.g. temperature variations) not reflected in the current ECIR estimate.

This remainder is used in [1] as an estimate error to update the ECIR in a frequency-domain Least Mean Square (LMS) algorithm [6]. The LMS update (correction term) is a product of the complex conjugate of the frequency domain representation of the transmitted symbol scaled by the chosen step size and the estimation error. The convergence of this algorithm was shown in [1]. The computational complexity is one complex multiply and add per subchannel, which translates to 9.5 MOPS for the central office and 5.7 MOPS for the remote terminal, assuming that updates are performed for every data symbol at a standard rate of 4000 symbols/s.

The primary shortcoming of the method proposed in [1] is the presence of the far-end signal in the estimation error. The presence of the far-end signal raises the mean square error (MSE) of the ECIR LMS updates. The increased MSE (1) masks small changes in the ECIR, thus reducing the performance of the receiver over time; (2) makes the LMS update less stable [6]; and (3) reduces the ability of the algorithm to respond quickly to changes in the ECIR. The farend signal may also contain subchannels with constant content (i.e. pilot tone), which become a part of the estimation error. The far-end signal is a source of noise for the ECIR LMS updates. The poor convergence of the LMS update in the presence of high noise levels is well known [6].

The far-end signal-to-echo ratio decreases with the length of the twisted-pair copper loop. The influence of the far-end signal in the LMS update will be stronger on a shorter twisted pair copper loop than on a longer loop due to reduced channel attenuation.

\section{PROPOSED ALGORITHM}

The previously proposed ADSL EC architecture and algorithm in [1] exploited the FFT and inverse FFT (IFFT) in the ADSL DMT transceiver architecture, and used a frequency-domain LMS algorithm for updating the EC filter coefficients. Our proposed improvement eliminates the farend signal from the LMS estimation error while maintaining the LMS updating structure. The update is performed using the estimation error containing only the true residual echo. This reduces the MSE of the LMS algorithm by the second moment of the far-end signal. Thus, for the same step size of

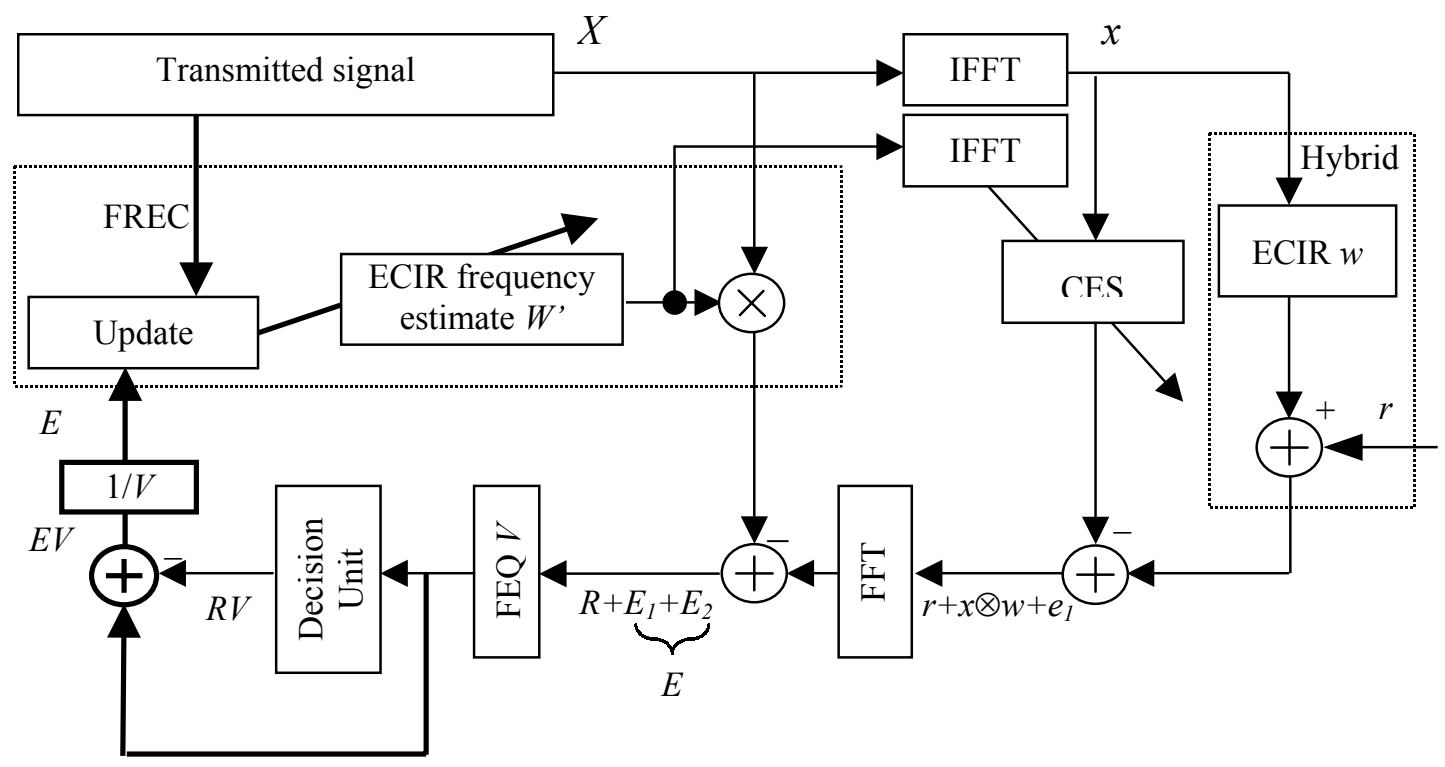

Figure 1: Remote terminal echo cancellation system with EC tap updates (the bold lines indicate new blocks compared to the method in [1]) 
Echo

\begin{tabular}{|c|c|c|c|c|c|c|c|c|}
\hline & $\mathrm{Sym}$ & ol $n$ & $\mathrm{GP}$ & $n+1$ & $\mathrm{QP}$ & $n+2$ & $\mathrm{QB}$ & $n+3$ \\
\hline $\mathrm{GP}$ & Symbol $n$ & 6 & $n+1$ & $\ddot{C}$ & $n+2$ & $\mathrm{QP}$ & $n+3$ & \\
\hline
\end{tabular}

Figure 2: Misalignment of the echo and the far-end signal on the central office side where CP stands for cyclic prefix

the LMS iteration, our method converges faster than the method in [1]. Remote terminal and central office architectures are discussed separately below.

This approach is new for the ADSL technology. However, elimination of the far end signal has been explored by Falconer [7] for a full-duplex two-wire digital transmission on digital subscriber loops. Falconer shows that the adaptive reference echo cancellation (AREC) algorithm based on decision-based estimation and removal of the far end signal offers much faster convergence and shorter coefficient word lengths than the algorithms without far end signal removal.

\section{A. Remote Terminal Architecture}

A block diagram of the proposed system architecture is shown in Fig. 1. The transmitted frequency-domain symbol $X$ is transformed into a time-domain symbol $x$, which passes through the ECIR and is added to the far-end signal $r$. After the time-domain processing unit CES, the linear convolution of $x$ and $w$ (ECIR) is converted into $x \otimes w+e_{1}$ where $\otimes$ denotes circular convolution and $e_{1}$ is the error incurred during this operation due to the imperfect estimate of the ECIR in the CES. The signal $r+x \otimes w+e_{I}$ becomes $R+X W$ $+E_{l}$ in the discrete-frequency domain. After the Frequency Domain EC (FREC) unit, the estimate of the echo is subtracted out, which leaves $R+X\left(W-W^{\prime}\right)+E_{1}=R+E_{2}+$ $E_{I}=R+E$, where $W^{\prime}$ is the current frequency-domain estimate of the ECIR. This signal consisting of the far-end signal and residual echo is fed into the Frequency Domain Equalizer (FEQ) rather than fed back directly to the FREC as was proposed in [1]. The FEQ $(V)$ is a frequency-domain filter containing an estimate of the inverse of the transmission channel frequency response ${ }^{1}$ and it changes the magnitude and phase of $R+E$ to produce $(R+E) V$. The decision unit outputs the received constellation vector ${ }^{2}, R V$, with the assumption that the noise levels (residual echo and transmission channel induced noise) are low enough so that the decision unit can correctly decode the its input.

ADSL systems employing echo cancellation have an initial training period during startup that produces the initial ECIR estimate. The LMS starts the updates from this initial estimate. The initial ECIR coefficients provide a good initial estimate of

\footnotetext{
${ }^{1}$ This paper assumes that the FEQ block is perfectly matched to the channel impulse response. In reality, the FEQ will not be perfectly matched. ADSL transceivers commonly adapt FEQ taps to improve the match with the channel impulse response.

${ }^{2}$ This paper assumes that no other noise is present in the system besides the residual echo. In reality, the channel induces noise and this noise will be a part of $E V$.
}

the ECIR so the initial residual echo is significantly smaller than the far-end signal and does not cause erroneous slicing in the decision unit. Given that the changes in the ECIR are not large and the LMS updates are tracking the changes, the residual echo will also not cause erroneous decisions.

Subtraction of the decision unit's output $R V$ from its input yields the residual echo $E V$, which is free of variation and bias due to the far-end signal but modified in magnitude and phase by the FEQ coefficients $V$. Because the FEQ is not a part of the echo channel, the inverse operation $1 / V$ must be performed to obtain $E$. This operation is computationally expensive and can be replaced with an approximate division operation [8]. The recovered $E$ may now be applied to the FREC to perform the LMS update. The additional blocks come at a cost of only one additional complex add and one complex multiply per subchannel compared to the method in [1]. Assuming that the update is performed every symbol and for every subchannel at a rate of $4000 \mathrm{symbols} / \mathrm{s}$, the increase in computational complexity is approximately 6.1 MOPS over [1]. All of the other blocks are already a part of a remote terminal.

\section{B. Central Office Architecture}

On the central office side, we again remove the far-end signal from the estimation error, thus rendering the update independent of the far-end signal. The central office transceiver, however, faces a time misalignment between the transmitted and far-end data symbols (Fig. 2). Because of this time misalignment, which does not occur in the remote terminal, the central office transceiver performs EC entirely in the time domain. For further details on symbol misalignment issues, the reader is referred to [1] and the ANSI standard $\mathrm{T} 1.413$ [3].

The block diagram of the proposed system architecture is shown in Fig. 3. The far-end signal can be removed from the estimation error only after the decision unit performs slicing in the frequency domain. Both the FFT and the decision unit operate on one symbol of the far-end signal at a time. Due to symbol misalignment and the fact that the FFT is synchronized to the far-end signal and not to the echo signal, the result after the removal of the far-end symbol is not an estimation error symbol that can be used in the LMS update. The residual echo symbol at index $n$ is 'pieced together' out of at most two received signal symbols, at indices $n$ and $n+1$. In Figure $3, e_{n}$ and $e_{n+l}$ denote parts of the residual echo symbol at index $n$. The samples of the estimation error symbol that overlap with the $\mathrm{CP}$ of the far-end signal cannot be recovered. One possible solution zeroes out those estimation error 
samples, and another uses interpolation. $\mathrm{CP}$ is only 4 samples long at the central office and the choice of either of the schemes will not produce large differences in the frequency
The simulation results given in Fig. 4 demonstrate convergence of the ECIR coefficients to the true pre-set ECIR in the course of LMS updates. The initialization of the FREC

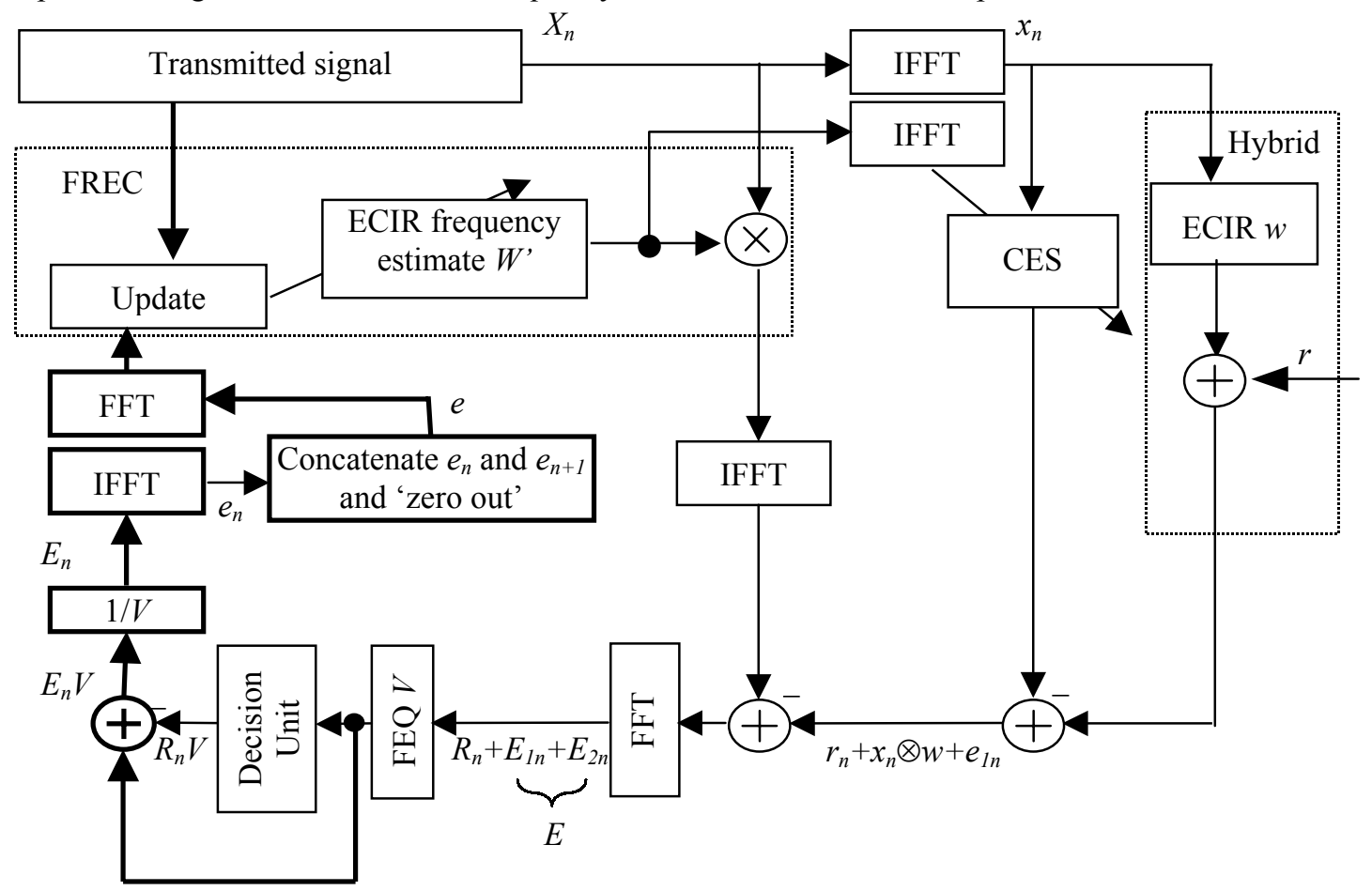

Figure 3: Central office echo cancellation system with EC tap updates (the bold lines indicate new blocks compared to the method in [1])

domain of residual echo. We choose to zero out the residual echo samples because of the lower computational complexity. Because of the symbol misalignment, the extraction of the residual echo symbol has to occur in the time domain, hence the need for an IFFT and a later FFT in the residual echo signal path. After the residual echo symbol is assembled, the LMS updating mechanism is the same as it was at the remote terminal.

The additional blocks require one complex add and one complex multiply per subchannel and two FFT/IFFT operations per symbol over the method in [1]. Given that the update is performed for every symbol and for every subchannel at a rate of $4000 \mathrm{symbols} / \mathrm{s}$, the increase in computational complexity over the method in [1] is approximately 2.3 MOPS. Note, that the increase in computational complexity at the central office is less than at the remote terminal despite two extra FFT/IFFT operations because there are $1 / 8$ as many carriers to process in the central office compared to the remote terminal.

\section{EXPERIMENTAL RESULTS}

We simulated the remote terminal and central office architectures in MATLAB and implemented them in a commercial VLSI ADSL Transceiver. Due to the limitations posed by symbol misalignment, the implementation on the central office side is more difficult compared to the remote terminal and puts the algorithm through a more difficult test. coefficients was preformed in the course of the ADSL transceiver initialization as given by G.DMT over the standard carrier area serving (CSA) loop 7 [3]. The EC coefficients were then offset from their initialization value (reference EC coefficients) and the performance of the EC updating mechanism was observed for the proposed method and for the algorithm in [1]. The variance of the additive white Gaussian noise used was $-140 \mathrm{~dB}$, the transmission channel used was the standard carrier area serving (CSA) loop 7, the power of the received far-end signal was $-61 \mathrm{~dB}$, and the power of the echo signal was $-26 \mathrm{~dB}$.

As the physical ECIR was the same throughout the experiment, the success of the updating algorithm could be measured by the speed at which the EC coefficients converge back to the reference EC coefficients. The proposed algorithm converges within $3 \mathrm{~dB}$ of the true taps in 200 iterations while the algorithm proposed by $\mathrm{Ho}$ et al. takes around 800 iterations to accomplish the same task. The experiments showed that the presence of the far-end signal in the estimation error slows the rate of convergence of the estimate (Fig. 4a) vs. the proposed algorithm when the far-end signal has been removed (Fig. 4b).

\section{CONCLUSION}

In this paper, we propose an efficient echo cancellation method for DMT based systems. This method eliminates the dependence of the updating mechanism on the far-end signal. 
This reduces the estimation error present in LMS iterations thus enabling the echo canceller to adapt faster and better to the changes in the modeled signal path. The improvement in the performance of the proposed EC updating algorithm also
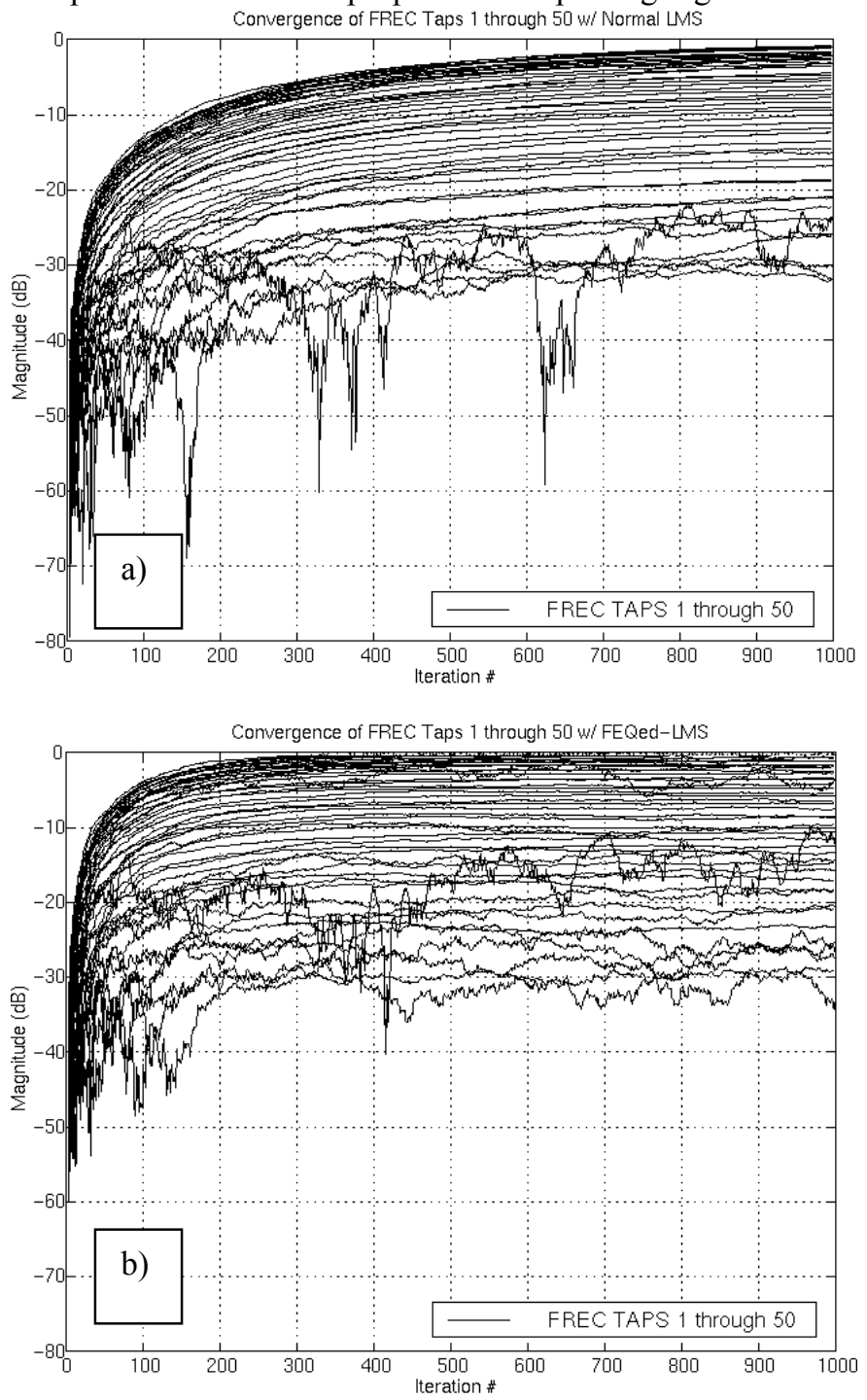

Figure 4: Convergence of the magnitude of the frequency domain echo canceller coefficients 1-50 for the LMS updates that include far-end signal (a) and the LMS updates that do not contain far-end signal (b). A set of echo canceller coefficients that corresponds to the given ECIR is used as a reference and convergence of both (a) and (b) is measured against this known set. requires $64 \%$ more computations for the central office and $40 \%$ more computations for the remote terminal transceiver compared to the EC updating algorithm in [1].

\section{ACKNOWLEDGMENT}

The authors would like to thank Dr. Lucio F. C. Pessoa and Mr. Jaksa Djordjevic at Motorola in Austin, TX and Dr. Guner Arslan at Cicada Semiconductor in Austin, TX for their help in editing of this paper.

\section{REFERENCES}

[1] M. Ho, J. M. Cioffi, and J. A. C. Bingham, "Discrete Multitone Echo Cancellation," IEEE Trans. Commun., vol. 44, pp. 817-825, July 1996.

[2] J. M. Cioffi and J. A. C. Bingham, "A Data Driven Multitone Echo Canceller," IEEE Trans. Commun., vol. 42, pp. 2853-2869, Oct. 1994.

[3] ANSI "Network and customer installation interfaces: Asymmetric digital subscriber line (ADSL) metallic interface," in American National Standard for Telecommunications, vol. T1E1.413, 1998.

[4] ITU-T “Asymmetric Digital Subscriber Line (ADSL) Transceivers," Int. Telecommunications Union, Draft Recommendation G.992.1, June 1999.

[5] ITU-T "Splitterless Asymmetric Digital Subscriber Line (ADSL) Transceivers," Int. Telecommunications Union Recommendation G.992.2, June 1999.

[6] S. Haykin, Adaptive Filter Theory, 3rd Ed., Prentice Hall, Englewood Cliffs, NJ, 1996.

[7] D. D. Falconer, "Adaptive Reference Echo Cancellation," IEEE Trans. Commun., vol. 30, pp. 2083-2094, Sep. 1982.

[8] M. Milosevic, P. Molnar, T. Inoue, and M. Pendleton, "Method and Apparatus for Echo Cancellation Updates In a Multicarrier Transceiver System," U. S. Patent pending, March 2000. 